\title{
Environments for Sonic Ecologies
}

\begin{abstract}
This paper outlines a current lack of consideration of the environmental context of Evolutionary Algorithms used for the generation of music. This paper attempts to readdress this balance by outlining the benefits of developing strong coupling strategies between agent and environment. It goes on to discuss the relationship between artistic process and the viewer and suggests a placement of the viewer and agent in a shared environmental context to facilitate understanding of the artistic process and a feeling of participation in the work. The paper then goes on to outline the installation 'Excuse Me' and how it attempts to achieve a level of Sonic Ecology through the use of a shared environmental context.
\end{abstract}

Keywords: Evolutionary Music, Ecology, Environment, Installation, Agent, Artwork.

\section{Introduction}

Within the field of Artificial Intelligence there has been a relatively recent embracement of Ecological Theory [1], with most research now centering on the importance of an agent's embodiment within an environment and the consequential contribution to the growth and development of a situated definition of cognition. This ecological approach moves away from the classical idea of a dualism of mind and body [18] towards a philosophy where the interaction between the agent and its environment constitutes a reciprocal relationship which is defined by the agents' physical embodiment within an environment and its ecological relationship to it.

Despite a long tradition of site specific composition in music, with examples as far back as the Baroque (Gabrielli, Bach [21]); developments of concepts of space and situatedness in composition (Mozart, Serenade in D for 4 Orchestras (K286 1777), Stockhausen, Gruppen for 3 Orchestras 1955,[21]); the later obsession with multichannel speaker transmission by electroacoustic composers [19] and the embracement of a concept of the environment by groups such as 'The World Soundscape Project', founded by Schafer [20] and publications such as Truax's 'Acoustic Ecology' [23], it is only recently that theorists and artists such as Whitelaw [25] and McCormack [15][14] have started calling for a similar level of environmental consideration in systems for the generation of sound/music that utilise models from the field of Artificial Intelligence or more specifically Evolutionary Computation. 


\section{The Un(der)-modeled Environment}

As John McCormack stated in his 2006 article 'New Challenges for Evolutionary Music and Art', '[o]ne common oversight made by those trying to evolve creative systems is proper consideration of environment' [15]. Systems taken from the field of Evolutionary Computation applied to the generation of music tend to themselves be borrowed from models inspired by systems found in nature. These models especially those based on 'non-reductionist' techniques tend to consist of varied numbers of agents existing within a shared virtual environment. Programmers determine not only the nature and strength of the interactions between the individual agents but also the quantity and form of any environmental reciprocity. The strength of environmental coupling can vary from weak to strong but has a tendency to fall into the former category, possibly due to computational limitations and the corresponding desire to keep everything as simple as possible. Whitelaw turns to emergent swarms as an example of how agents can be modeled so removed from their environment; although they interact with each other within the programmed space, this space is static, indifferent to the plight of its occupants. An agent in a swarm is described as a 'clone in a crowd, unchanging, no traction of the space it inhabits, existing in an ongoing perpetual present' [25]. Some recent environmental models [6][7][22] have included environmental factors such as food sources, disease and even evolving critics and this deeper relationship between environment and agent seems to give an 'aesthetic and generative payoff' [25]. Yet a truly reciprocal relationship between agent and environment seems to be lacking. More thought therefore needs to be given to the process of embodiment of the agents within their environment and the level of environmental coupling that is afforded to them. How are their interactions to be designed and handled? For a deeper relationship between agent and environment, one in which there is a level of mutual exchange between the two; where environmental constraints can lead to unexpected outcomes; account for dynamic fitness landscapes and for the development of unforeseen or unexpected niches, can generate a climate for the generation of a society based on interaction rich in complexity and creativity.

\section{Embodiment}

\subsection{The Embodied Agent}

'The affordances of the environment are what it offers the animal, what it provides or furnishes either for good or ill' [9].

Gibson postulates that our relationship with the environment is governed by our perception of it, which in turn is dependent on our bodily interactions within it. Different encounters in different environmental situations afford different qualities to different beings through interaction with their different bodies. Thus the way in which we perceive our environmental context is directly linked to our embodiment within it. 
Thus embodied perception of our environment is not just a case of situated experience, but rather an example of the deeper relation your body has on the understanding of the environment. For example, water for a pond skater affords a supportive surface where as for a human, due to its heavier body mass, it does not.

Interpretation of the environment is thus directly linked to an agent's embodiment within it, not just its level of embodiment physically, but what these sensed perceptions afford within its environmental context. Not only are agents situated in a dynamic environment, but also the way they perceive this environment is dependent on a combination of their embodiment within it and their bodily interpretation of it. The environment can thus be considered as a dynamic landscape within the system, possibly even as a dynamic agent in its own right. Environmental constraints themselves can be considered as an evolving fitness landscape with which the agents interact, thus allowing for a co-evolution of agent and environment [16] describing a complex relationship that never reaches a stasis.

\subsection{The Embodied Viewer}

A description of a viewer's relationship with a generative artwork, can be a controversial one. There is one stance that suggests that the viewer's appreciation of the work comes about through an understanding of the beauty of the generative algorithm [4]. Other theories are that Evolutionary Art holds the attention of the viewer through comparability with models from nature, [10] for others it is an emergence of apparently creative output from a computer that draws them in. Either way there is either a retinal [8]/sensory [12] or rational/cognitive [10] perception of beauty. Thus in both cases there is a strong relationship with how the viewer perceives the work (retinal), or how much of the process they perceive (cognitive) and what they get out of the work. Rather than considering the viewer as a voyeur, outside of the artistic process, listening in, removed from what is happening, looking down upon a virtual world as a kind of god like creature, omnipresent but impotent, exercising the notion of aura, described by Walter Benjamin as essential for the perception or art [3] What if instead of this the viewer is embodied within the same environmental constraints and space as the agents? In this scenario, due to a shared locality of stimuli an understanding of the system space through sensual exploration becomes attainable. Rather than advocating a deeper level of immersion of the voyeur into the virtual world one could argue that this sharing of system-space is best achieved by introducing the agents into the same world as the perceiver i.e. the 'real' world. This facilitates a greater immersion in the piece with higher levels of communicability between viewer and agents, viewer and process and hopefully 'viewer' and music. The viewer can now be considered a participant in the systemspace of the installation, another agent in the system. There is no need for a model a virtual environment as the real world is an environment in its own right with more inherent richness that can be found in any simulation. This sharing of environmental context not only facilitates a direct communication between agent and participant but 
also encourages an ecology of communication to develop where an evolution of agent and environment includes the participant as an active member of society.

\section{Enaction}

The enactive [24][17] viewpoint builds on Gibson's ideas of 'direct perception' by doing away with the need for a Cartesian inner world model or representation. Rather than the more traditional perception - computation - action model there is now a more direct relationship between perception and action, with action informing the perception, which guides action, which informs the perception etc. [5]

This idea of a lack of an 'inner world view' or 'representation' such that all computation is a result of a direct relationship between action and perception has a commonality with the idea of agents and participants coexisting in real space. In both situations there is a move away from complex representations in simulated space to all interaction and perceptual representation happening in the real lived environment. This facilitates a shared mode of perception between agent and participant and affords the participant an enactive approach to the perception of the generative process; all interaction is no longer modeled but happens through embodiment in a shared environment. The environment becomes the main catalyst for driving communication and creativity, each agent contributing to and taking from it like a shared cultural experience.

\section{5 'Excuse Me': A Vehicle for Enactive Exploration}

Installation is a medium that lends itself to an investigation of the viewer as an active participant within a shared system-space, another agent in the algorithm. There is already a concept in the medium of installation that the viewer is more than a voyeur, abstracted from the work. 'The term 'looking' is superceded in installation by the concept of 'spectating', which assumes a higher involvement by the audience' [2]. The installation 'Excuse Me' (2006), an interactive sound installation developed by the author, attempts to set up a situation in which the notion of spectating in installation is superseded by this notion of participation in the piece, to such an extent that the spectator is not only interacting with the piece but becomes another agent in the artwork, an active participant in the creative process.

'Excuse me' tries to create the conditions for a deeper relationship between agent, participant and environment to develop. One in which there is a level of mutual exchange between the agents (including the participant) and environment; where environmental constraints can lead to unexpected outcomes; can account for dynamic fitness landscapes and for unforeseen niches to develop. For it is this relationship, 
formed on deeper embodiment of agents within their surroundings, with which we hope to generate a climate for richer results of complexity and creativity.

In 'Excuse me', the agents are placed in the same environment as the participants; they can communicate with this environment through listening and speaking devices, microphones and speakers. Allowing the participant and agents to coexist in the same environment and sonic ecology. Participants can have a direct affect on the ecology of the piece through sonic and physical interaction through an individual agent or with the environment as a whole.

\subsection{Installation Design}

Each agent consists of a speaker and microphone element. The speaker element is constructed out of an audio transducer attached to the body of a violin; the microphone is a homemade electret with attached preamp. These microphones are placed somewhere on the body of the violin. There are six agents situated in the installation space, suspended from the ceiling, six being the minimum number considered by the author to be needed to form a society of sonic interaction. They are spaced apart with room for people to move amongst them and interact with them as individuals. The six agents listen to their environment and analyse the audio input to their microphone using Tristan Jefran's Max/MSP object, analyzer [11]. They then try to recreate their incoming aural stimuli through a matching process of this analysis of this audio to their own internal database of sounds. The microphone and speaker device of each agent is connected to a computer running Max/MSP [13]. After the agent has played back its 'best fit' sound to the environment it adds the analysis of the incoming sound to its internal database.

This re-sharing of an agent's interpretation of the sound back into the environment allows for a culture of communication to develop, where the agents build up a library of shared sonic experiences. This kind of mimetic interaction is easy for the participant to appreciate and partake in. The algorithmic process is somewhat transparent and is only affected externally by environmental factors that are common to both agents and participant.

\subsection{Subversion of Intention}

A subversion of the mimetic intention of the agents is employed as a catalyst to the development of the sonic output. The system of analysis and recreation employed by the agents does not always give predictable results. For example the pitch-detecting algorithm is best suited to working in non-noisy, clean environments on monophonic sounds. Asking it to detect pitch of sound from a noisy microphone plugged into the environment, not only detecting one agent's polyphonic output but an unpredictable complex output from up to five other agents at once, plus possibly other extraneous environmental sounds is pushing the software far beyond its intended limits. What do 
these machines make of the noisy surroundings they have been placed in, how will they interpret these situations where they are experiencing information overload? This added complexity is a benefit from the fact that the communication of these agents and their perceptual reconnaissance is all carried out in a real unpredictable environment, which leads to an evolution of un-predictable outcomes that could in turn be labeled creative.

Allowing the viewer to walk amongst these agents, listening to their individual output or listening to the society as a whole, whilst interacting on many different levels helps them to understand the internal (now external) algorithmic process. The agents pick up their environmental noise and try to recreate it; bits of speech and other sounds are broken up and reinterpreted, passed from one agent to another. Creating an evolving shared language of communication, a sonic ecology, an emergent texture of sound.

\section{Conclusion}

Through notions such as embodiment and enaction one can revisit design strategies for algorithms that pertain to operate in a creative environment. We've argued that it's worth exploring strategies in which emergent and evolutionary algorithms inhabit an environment that is shared by a viewer/participant. We have questioned the role of the algorithm as model for delivering creative solutions directly and have proposed that both the algorithm and the viewer need to be situated and embodied in a shared environment. As exemplified in the installation "Excuse Me", there is no attempt at creating a reductionist model of the environment context but rather a placing of the algorithm and the viewer within the full complexity of the environment as a way of promoting unexpected complexities and interactions.

\section{References}

1. Almeida e Costa,F. \& Rocha, L.M.: Embodied and Situated Cognition. Artificial Life. Vol 11 (1,2) MIT Press. pp 5 -11. (2005)

2. Benjamin,A (ed.): Installation art. Art \& design profile ;no. 30. Art \& design ;vol.8, no.5/6. London : Academy Group, (1993)

3. Benjamin, W.:The Work of Art in the Age of Mechanical Reproduction. 1935. Zeitschrift f ü $\mathrm{r} \quad \mathrm{S}$ o z i a l f ors chung (o riginally). www.marxists.org/reference/subject/philosophy/works/ge/benjamin.htm Accessed online (30 October 2006)

4. Borevitz,B.: Super-Abstract: software art and a redefinition of abstraction. read_me Software Art and Cultures Edition, Goriunova,O \& Shugin,A [eds] Center for Digital AEstetik-forskning, 2--4 pp 310-311. Also available online http://runme.org/project/+super-abstract/ accessed 29/10/06 
5. Clark, A. :From Fish to Fantasy: Reflections on an Embodied Cognitive Science. A shortened and amended version appears as "An Embodied Cognitive Science?" Trends In Cognitive Sciences 3:9:1999 pp 345-351. 1999 also online http://www.philosophy.ed.ac.uk/staff/clark/publications.html accessed 29/10/06

6. Dorin, A.: Beyond Morphogenesis: enhancing synthetic trees through death, decay and the Weasel Test, in Third Iteration: Proceedings of the Third International Conference on Generative Systems in the Electronic Arts, Innocent, Brown, McCormack, Mcilwain (eds) CEMA, Melbourne, Dec. (2005), pp119-128

7. Dorin, A.: The Sonic Artificial Ecosystem in Proceedings of the Australasian Computer Music Conference (ACMC), Haines (ed.). Adelaide, Australia. (2006). pp32-37

8. Duchamp,M. \& Cabanne,P.: Dialogues With Marcel Duchamp. Da Capo Press. New York. (1987)

9. Gibson, J,J.: The Ecological approach to visual perception. LEA, London. (1986).

10. Jaschko. S.: Process as aesthetic paradigm: a nonlinear observation of generative art. generator.x conference, 23 - 24.September 2005, Oslo, Atelier Nord, (2005).

11. Jehan, T.: analyzer for Max/MSP available from http://web.media.mit.edu/ tristan/ accessed online on 29/10/06

12. Kant, I.: Observations on the Feeling of the Beautiful and Sublime. Trans. John T. Goldthwait. University of California Press, 1961, (2003).

13. Max/Msp : http://www.cycling74.com accessed online on 29/10/06.

14. McCormack, J. : New evolutionary challenges for evolutionary music and art. Lanzi, P.I [ed] ACM SIGE V Olurion Newsletter Vol 1 (1) April 2006 pp 5- 1. (2006)

15. McCormack,J: Open problems in Evolutionary music and art. Proceedings of EvoMusart 2005, 30th March to 1st April Lausanne, Switzerland. (2005)

16. Moreno, A. \& Etxeberria A. :Agency in natural and artificial systems Artificial Life Vol. 11 Issue 1-2 pp 161-176. (2005)

17. Noe, A.: Art as Enaction accessed online http://www.interdisciplines.org/artcog/papers/8/version/original (14/08/06)

18. Rozemond, M.: Descartes's Dualism. Cambridge: Harvard University Press (1998).

19. Smalley,D.:The Listening Imagination: Listening in the Electroacoustic Era. Contemporary Music Review 13:2. (1997).

20. Schafer,R.M. (1994) The Soundscape: our sonic environment and the tuning of the world. Destiny Books, Vermont. (originally pub 1977). (1994).

21. Stevenson, I.: A Dialetic of Audible Space available online http://www.headwize.com/articles/steven_art.htm accessed on 29/10/06

22. Todd, P.M. \& Werner, G.M.: Frankensteinian Methods for Evolutionary Music Composition. Griffith, N., and Todd, P.M. (Eds.) (1998). Musical networks: Parallel distributed perception and performance. Cambridge, MA: MIT Press/Bradford Books. 1998

23. Truax B. (2000).: Acoustic Communication, Greenwood Press, Westport, Connecticut 2nd edition. (2000) (1st Edition. Ablex Publishing, 1984)

24. Varela, F.J., Thompson, E. \& Rosch, E..: The embodied mind : cognitive science and human experience. London; Cambridge, Mass. MIT Press. . (1991).

25. Whitelaw,M. (2005) System Stories and Model worlds: A Critical Approach to Generative art.. Readme 100 : Temporary Software Art Factory. (Norderstedt: BoD) pgs 135-154 Dec (2005). 